\title{
A General Method to Label Metal Oxide Particles with Fluorescent Dyes Using Aryldiazonium Salts
}

\author{
Jean-François Dechézelles, Nébéwia Griffete, Hervé Dietsch, and Frank Scheffold*
}

\begin{abstract}
Fluorescent particles are widely used in different research arenas in biology, physics, materials, and nanoscience. In targeted therapeutics or cellular imaging, fluorescent particles have been an essential factor in driving major scientific advances over the last decades. ${ }^{[1-3]}$ In physics, fluorescent particles act as tracers to study Brownian motion or diffusion behavior in complex fluids or particle dispersions, ${ }^{[4]}$ while in material science they are widely employed not only for coloring and paints but also for harvesting solar energy in dye-sensitized cells. $^{[5]}$
\end{abstract}

Different methods are applied to attach fluorescent dyes to the surfaces of small particles. Some dye-labeling protocols are efficient and well established such as the hydrolysis and condensation of functional silane agents onto a silica $\left(\mathrm{SiO}_{2}\right)$ surface and subsequent silane compounds anchoring. ${ }^{[6]}$ Using a dye-labeled monomer and a posteriori modification of the polymer chains are also standard procedures for dyelabeling polymer nano- and microbeads. ${ }^{[7,8]}$ A variety of such fluorescent particles are also commercially available. General concepts for dye-labeling particles with different surface chemistry remain however scarce. A notable exception is the layer-by-layer assembly of (dye-labeled) polyelectrolytes, which however has the major drawback of being tedious and timeconsuming due to the many deposition and purification steps required. ${ }^{[9,10]}$

Methods toward fluorescent labeling of metal oxide particles are currently restricted to a few special cases, while a general method for dye labeling this class of materials is still lacking. ${ }^{[11,12]}$ Considering its importance, an easy and versatile toolkit for dye labeling is thus urgently needed. Especially, pressing is the need for dye-labeling concepts for metal oxide particles, such as titanium dioxide $\left(\mathrm{TiO}_{2}\right)$ or gadolinium oxide $\left(\mathrm{Gd}_{2} \mathrm{O}_{3}\right)$, for their high refractive index or magnetic properties, that cannot be addressed by traditional approaches. This is unfortunate since $\mathrm{TiO}_{2}$ and other metal oxide particles are among the most widely used nanoparticle (NP) species with

Dr. J.-F. Dechézelles, Dr. N. Griffete, Prof. F. Scheffold

Department of Physics and Fribourg

Center for Nanomaterials

University of Fribourg

Chemin du musée 3, $\mathrm{CH}-1700$, Fribourg, Switzerland

E-mail: frank.scheffold@unifr.ch

Dr. H. Dietsch

Adolphe Merkle Institute and Fribourg Center for Nanomaterials University of Fribourg

Route de l'ancienne papeterie CP 209, CH-1723 Marly 1, Switzerland potential applications ranging from gene therapy, tumor imaging, materials science to nanotoxicity research. ${ }^{[5,11-14]}$ Moreover, such an approach could be used to dye $\mathrm{TiO}_{2}$ particles deposited onto the transparent anode of dye-sensitized solar cells. This, in turn, might allow the use of superior dyes, not yet commonly employed because of a non-adapted chemical functionalization. ${ }^{[5]}$

In the present work, we demonstrate fluorescent labeling of several metal oxide particles using aryldiazonium salts. To our best knowledge, most of these particles have never been fluorescently labeled by covalent linkage. Aryldiazonium salts are excellent candidates to achieve this goal since they have been previously shown to covalently react onto the surface of carbonbased and metallic surfaces. ${ }^{[15-17]}$ For example, as reported recently, Griffete et al. ${ }^{[17]}$ have postfunctionalized iron oxide NPs with a hydroxyl group using diazonium salt chemistry and Mesnage et al. ${ }^{[18]}$ have successfully grafted methacrylate polymer chains onto $\mathrm{TiO}_{2}$ particles. The main advantage of this strategy is that under well-defined conditions, chemical groups from aryldiazonium salts can spontaneously functionalize surfaces via the diazonium group transformation. This synthesis progresses rapidly at room temperature, involves only simple steps and moreover can be applied to many different types of inorganic surfaces. In contrast to many other functionalization methods, a covalent bond is established between the metallic surface and the organic layer during the grafting of diazonium salts. This has been demonstrated on metals by X-ray photoelectron spectroscopy (XPS), ${ }^{[19]}$ by time-of-flight secondary ion mass spectrometry (ToF SIMS), ${ }^{[20]}$ and density functional theory (DFT) methods, ${ }^{[21]}$ also on oxide surfaces several examples of grafting have already been published, for example, for indium tin oxide, ${ }^{[22]} \mathrm{SnO}_{2},{ }^{[23]} \mathrm{TiO}_{2},{ }^{[24]} \mathrm{CuO}^{[25]}$ In the latter, a $\mathrm{Cu}-\mathrm{O}-\mathrm{C}$ bond has been identified by XPS, indicating the existence of a covalent bond when diazonium salts are grafted on oxides.

In the present work, we demonstrate the great potential of this method by dye labeling several commercially available metal oxide particles such as $\mathrm{TiO}_{2}, \mathrm{Gd}_{2} \mathrm{O}_{3}$, and aluminum oxide particles. We note that our approach could easily be applied to organic particles as well and thus represents a general concept for the dye labeling of NP surfaces.

Using either one of two possible pathways (summarized in Figure 1), we couple a dye (rhodamine isothiocyanate, RITC) to an aryldiazonium salt (4-aminophenyldiazonium salt) via its amino group. We take advantage of the presence of the isothiocyanate group of the dye for initiating the addition reaction on the amino group of the phenyldiazonium. ${ }^{[6,26,27]}$ The first synthesis protocol consists of a two-step process: first the aminophenyl radical (activated by a basic $\mathrm{pH}$ ) is grafted onto the metal oxide NPs via the diazonium group. Second, the 


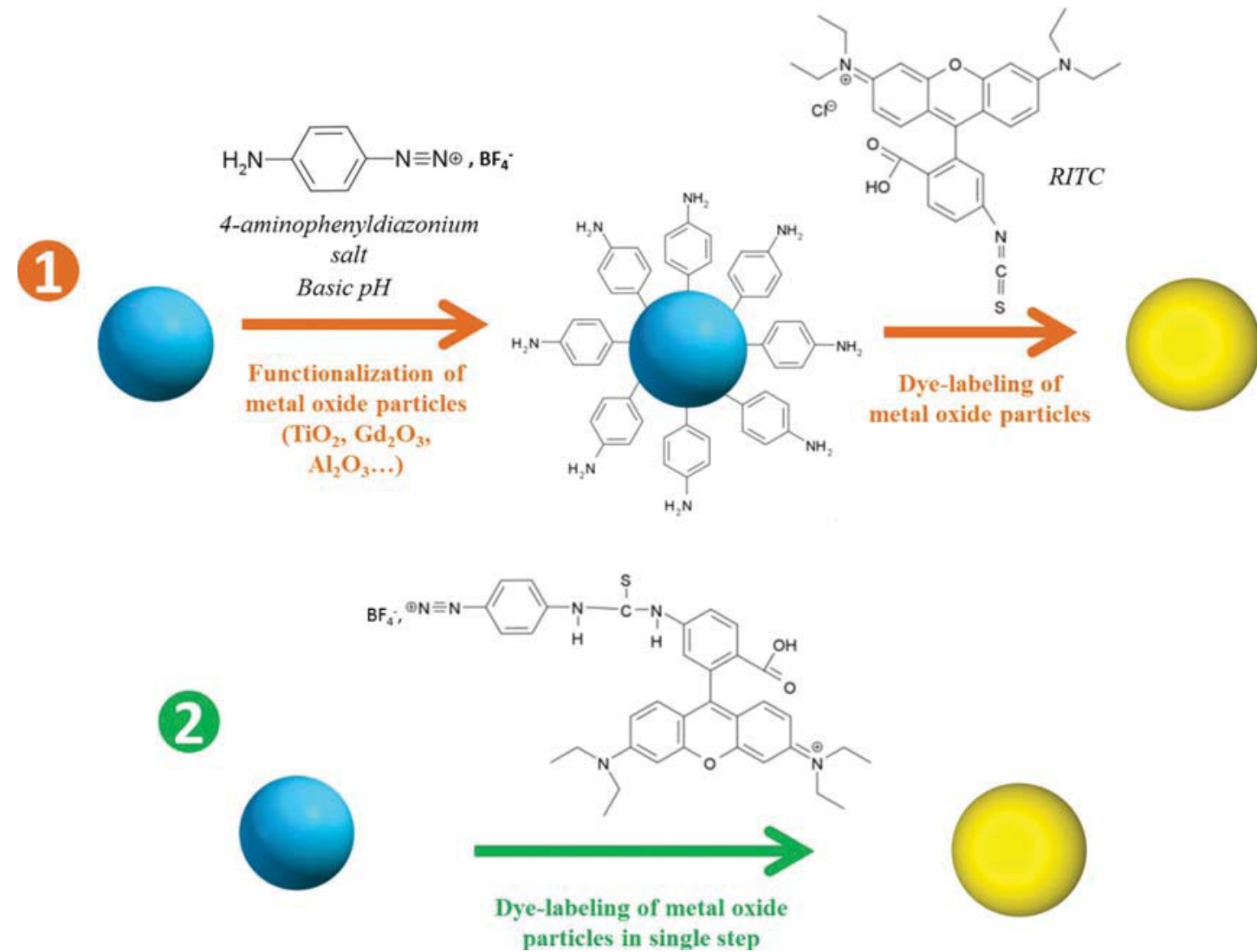

Figure 1. Schematic illustration of the two protocols used for dye-labeling metal oxide particles. In protocol 1, the aminophenyl radical (activated by a basic $\mathrm{pH}$ ) is first grafted onto the particle surface via the diazonium group. Then, the RITC reacts with the amino group to finally form a fluorescent particle. In protocol 2, fluorescent aryldiazonium molecules, which have been obtained by linking the aryldiazonium and the RITC, are grafted onto the particle surface.

fluorophore reacts with the amino group of the functional group present on the surface. The second pathway consists in grafting the fluorescent aryldiazonium molecule, previously obtained, by linking the aryldiazonium with RITC.

As a generic example for our labeling method, we discuss the case of micron-sized $\mathrm{TiO}_{2}$ particles. As explained above, the strategy for the dye labeling is based on two different protocols involving the addition reaction between the 4-aminophenyldiazonium salt and the RITC. In the first protocol, inorganic particles are initially covered with aryldiazonium salt to obtain a homogeneous aminophenyl coating of the surface. Then, the fluorescent shell around the particles is formed via the reaction between the dye and the amine group. To this end, bare $\mathrm{TiO}_{2}$ particles are transferred into a hydroethanolic mixture with a basic $\mathrm{pH}$ to facilitate the formation of the phenyldiazonium radical and to promote its attachment onto the $\mathrm{TiO}_{2}$ surface. The diazonium group of the molecule is first transformed in diazohydroxide, an intermediate form. The diazohydroxide is deprotonated with the alkaline $\mathrm{pH}$ and it forms a diazoate group that induces the molecule to become an aminophenyl radical, which can now react with the inorganic surface. ${ }^{[28,29]}$ Finally, we remove non-reacted aryldiazonium molecules by rinsing the functionalized particles several times with water and ethanol.

The second protocol involves the preparation of a fluorescent phenyldiazonium salt synthesized from the addition of the 4-aminophenyldiazonium with the RITC. A given volume of this fluorescent linking agent can be prepared and then later used for several labeling batches. For the $\mathrm{TiO}_{2}$ dye labeling, the dispersion $\mathrm{pH}$ is first increased to $\mathrm{pH} 9$ to promote the linkage with the $\mathrm{TiO}_{2}$ surface and subsequently the fluorescent agent is added to the dispersion. A centrifugation treatment is performed to remove the excess of non-reacted labeling molecules.

The chemical grafting of dyes can be discriminated by the disappearance of the diazonium group present in the infrared spectra after the synthesis of the diazonium salt. Figure SI-1 (Supporting Information) and Figure 2 allow comparison of the IR spectra of free $\mathrm{BF}_{4}, \mathrm{~N}_{2}-\mathrm{C}_{6} \mathrm{H}_{4}-\mathrm{NH}_{2}$, bare $\mathrm{TiO}_{2}$ particles and the products of the reaction between these two species, namely $\mathrm{C}_{6} \mathrm{H}_{4}-\left(\mathrm{CH}_{2}\right)_{2}-\mathrm{NH}_{2}$ labeled NPs. The final appears to be a combination of the spectra of pure NPs and of the aminophenyl moieties, confirming the presence of a $\mathrm{N}_{2}$ aminophenyl coating on the $\mathrm{TiO}_{2}$ particles. Yet, the most striking difference is the disappearance of the $\mathrm{N}=\mathrm{N}$ stretching mode near $2280 \mathrm{~cm}^{-1}$, indicating the release of $\mathrm{N}_{2}$ as a consequence of the cleavage of the diazonium moieties.

Next, we assess the success of our labeling reaction using ATR-IR. Figure 2 shows the IR spectra of the raw $\mathrm{TiO}_{2}$ materials and after each reaction step. The measured spectra are dominated by the contribution of the $\mathrm{TiO}_{2}$ particles because of the high volume ratio between the $\mathrm{TiO}_{2}$ and the organic molecules 


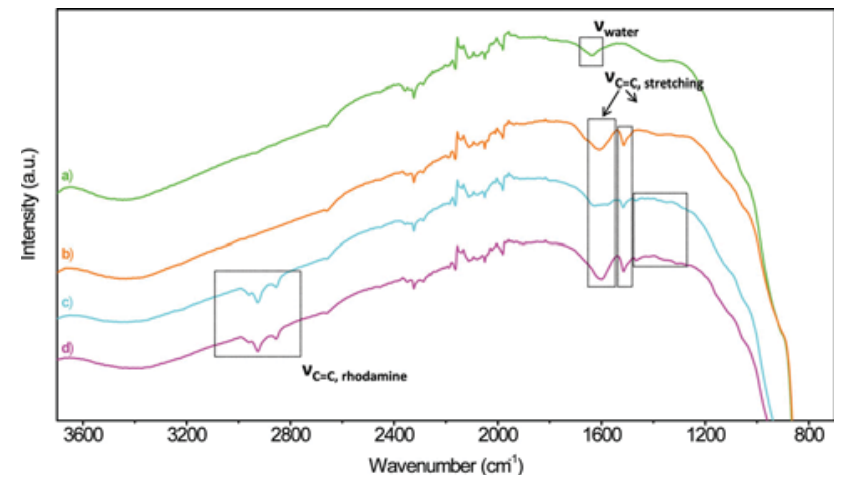

Figure 2. Attenuated total reflectance infrared spectroscopy (ATR-IR) spectra of a) $\mathrm{TiO}_{2}$ particles, b) phenyl- $\mathrm{NH}_{2}$-labeled $\mathrm{TiO}_{2}$ particles, c) phenylrhodamine-labeled $\mathrm{TiO}_{2}$ particles (protocol 1), and d) phenylrhodamine labeled $\mathrm{TiO}_{2}$ particles (protocol 2).

attached to the surface of the particles. As shown by Mesnage et al., ${ }^{[18]}$ the $\mathrm{TiO}_{2}$ particles absorb at $3430 \mathrm{~cm}^{-1}, 1640 \mathrm{~cm}^{-1}$, and strongly in the $800-600 \mathrm{~cm}^{-1}$ spectral region. These bands can be attributed to the absorption of water, the bending vibration of the $\mathrm{H}-\mathrm{O}-\mathrm{H}$ bonds of adsorbed water and the vibration of the $\mathrm{TiO}_{2}$ lattice. ${ }^{[18]}$ For the aminophenyl-grafted $\mathrm{TiO}_{2}$ particles, two additional bands appear at 1510 and $1600 \mathrm{~cm}^{-1}$ (Figure 2b). They can be attributed to aromatic $\mathrm{C}=\mathrm{C}$ stretching vibrations. ${ }^{[30]}$ In the spectral range $2400-3800 \mathrm{~cm}^{-1}$, we see no difference between the bare and the surface-functionalized particle. The evidence drawn from the IR spectra strongly suggests the successful covalent grafting of the molecules onto the surface of the inorganic particles. Finally, the surface-modified $\mathrm{TiO}_{2}$ particles are dye labeled by taking advantage of the addition reaction between the amino group of the diazonium and the isothiocyanate group of the dye. ${ }^{[6]}$ The RITC is covalently attached to the aminophenyl-labeled $\mathrm{TiO}_{2}$ particles via the 4-aminophenyldiazonium salt. Distinct differences now appear in the IRspectra of the aminophenyl-labeled $\mathrm{TiO}_{2}$ particles and the rhodamine-labeled $\mathrm{TiO}_{2}$ particles (Figure 2). In the $1200-1500 \mathrm{~cm}^{-1}$ and $2800-3000 \mathrm{~cm}^{-1}$ spectral regions, $\mathrm{C}=\mathrm{C}$ and $\mathrm{C}-\mathrm{H}$ stretching vibration bands can be directly related to the presence of the rhodamine dye on the surface of the inorganic particles.

Next, we apply the second, single step, labeling protocol (see Experimental Section) using the same batch of $\mathrm{TiO}_{2}$ particles. As shown in Figure 2c,d, the second dye-labeled $\mathrm{TiO}_{2}$ particle system displays essentially the same IR-spectral properties as the first one indicating again the success of the dye-grafting processes onto the inorganic surface.

In order to demonstrate the versatility of our approach, we apply both procedures, without any modifications, to several other interesting types of metal oxide particles such as aluminum oxide $\left(\mathrm{Al}_{2} \mathrm{O}_{3}\right)$, and $\mathrm{Gd}_{2} \mathrm{O}_{3}$. Moreover, as a proof of principle and for benchmarking the method, we also perform the grafting synthesis with standard $\mathrm{SiO}_{2}$ particles $(\varnothing \approx 700 \mathrm{~nm}$ ) obtained by the classic Stöber-Fink-Bohn method based on the hydrolysis and condensation of tetraethoxysilane (TEOS) in hydroethanolic medium with an alkaline $\mathrm{pH} .{ }^{[31]}$ For more detailed information, we refer to the Supporting Information (Figures SI-2 to SI-4). Finally, we perform a thermogravimetric analysis on bare and aminophenyl-grafted $\mathrm{SiO}_{2}$ particles to show the presence of the organic coating on the particles (see Figure SI-5, Supporting Information). A total weight loss of $7 \%$ is observed on heating phenyl-functionalized particles up to $800{ }^{\circ} \mathrm{C}$ while the uncoated NPs showed a weight loss of only $3 \%$ (probably due to some contamination species). This result suggests a high grafting density of organic molecules on the particle surface.

Finally, we assess the success of our synthesis by the analysis of real-space fluorescent microscopy images of the dye-labeled particles. A drop of a dilute suspension is applied onto a microscope cover slide. The images are taken with an inverted laser scanning confocal microscope (LSCM) (Nikon A1R) in a region where the solvent had evaporated in order to avoid image blurring due to Brownian motion. Titanium dioxide particles appear fluorescent under the LSCM (Figure 3a,b). For the labeled particles, we observe a maximum (inset Figure $3 \mathrm{a}$ ) of the emitted intensity at a wavelength of about $\lambda \approx 590 \mathrm{~nm}$, characteristic for the RITC dye used. Similarly the LSCM pictures of the $\mathrm{Gd}_{2} \mathrm{O}_{3}$ and $\mathrm{Al}_{2} \mathrm{O}_{3}$ particles (Figure $3 \mathrm{c}$,d) clearly demonstrate that the labeling process has succeeded.

In conclusion, we were able to demonstrate the covalent dye labeling of metal oxide particles using aryl diazonium salt chemistry. We use an amino-ended diazonium for the addition reaction with RITC before or after anchoring on the particles. The successful labeling of different types of metal oxide particles demonstrates the general use of our approach. Based on our results, we expect the application to many other types of particles, to be straightforward, and therefore the method
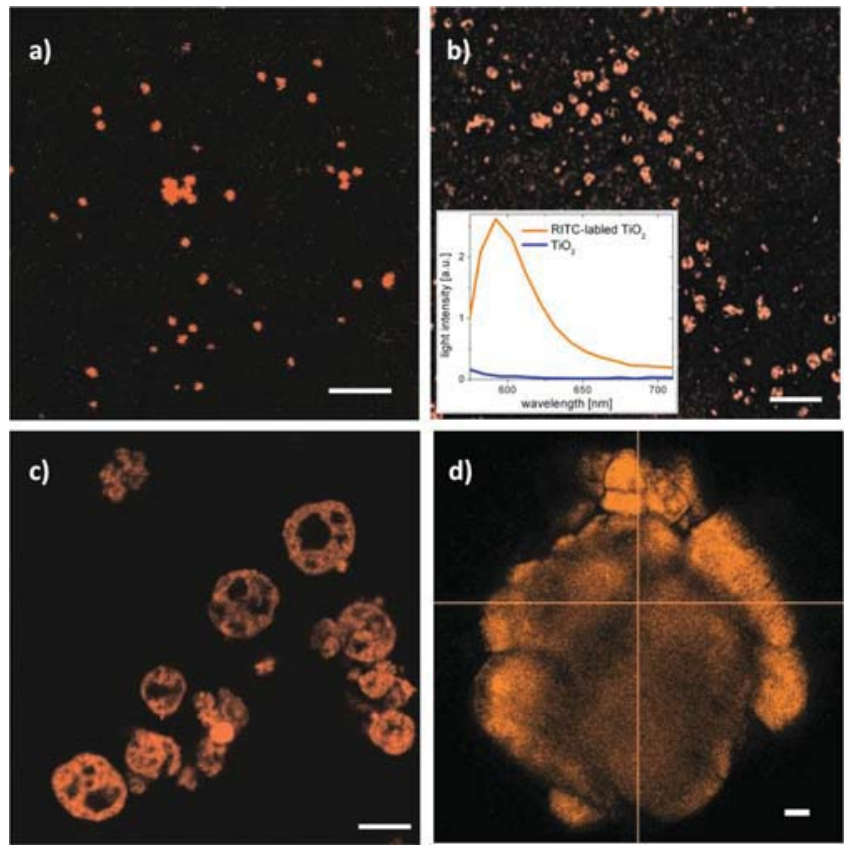

Figure 3. Confocal microscopy pictures of $\mathrm{TiO}_{2}$ particles labeled with RITC following the first protocol (a) and the second protocol (b), phenylrhodamine-labeled $\mathrm{Cd}_{2} \mathrm{O}_{3}$ particles (protocol 2) (c), and $\mathrm{Al}_{2} \mathrm{O}_{3}$ particles (protocol 2) (d). Scale bars are $5 \mu \mathrm{m}$. Inset: Measured wavelength dependence of the emitted light intensity recorded for RITC-labeled and bare $\mathrm{TiO}_{2}$ particles. 
should be of significant importance to a large number of research domains.

\section{Experimental Section}

Materials: Ammonium hydroxide solution, 25\% (Sigma-Aldrich), absolute ethanol (Honeywell), deionized water, and TEOS (GPR Rectapure, VWR Prolabo) were used for the $\mathrm{SiO}_{2}$ particles synthesis. Aluminum oxide particles (Merck), $\mathrm{Gd}_{2} \mathrm{O}_{3}$ particles (Sigma-Aldrich), $\mathrm{TiO}_{2}$ particles ( $99.9 \%$ pure, 325 mesh, CERAC) were used as supplied. Rhodamine isothiocyanate (mixed isomers, Sigma-Aldrich) was used for dye labeling. Phenylenediamine (Sigma), tetrafluoroboric acid (ACROS), and terbutylnitrite (Aldrich) were used for the synthesis of 4-aminophenyldiazonium tetrafluoroborate.

Synthesis of 4-Aminophenyldiazonium Tetrafluoroborate $\left(\mathrm{BF}_{4}^{-},+\mathrm{N}_{2}-\right.$ $\mathrm{C}_{6} \mathrm{H}_{4}-\mathrm{NH}_{2}$ ): To $3.71 \mathrm{~g}(34 \mathrm{mmol})$ of phenylenediamine, cooled at $0{ }^{\circ} \mathrm{C}$ in an ice bath, tetrafluoroboric acid was added dropwise to a total of $10 \mathrm{~mL}$ under continuous vigorous stirring. Next, $1.75 \mathrm{~g}(17 \mathrm{mmol})$ of terbutylnitrite was added and the reaction was left to proceed for $10 \mathrm{~min}$. The powder was subsequently dried and stored at $-5{ }^{\circ} \mathrm{C}$.

Functionalization of Metal Oxide Particles: $X \mathrm{mg}$ of metal oxide particles was added to a mixture of $1 \mathrm{~mL}$ of ethanol and $1 \mathrm{~mL}$ of water (see mass of metal oxide particles used in the following table). $200 \mu \mathrm{L}$ of $25 \%$ ammonia aqueous solution was introduced to increase the $\mathrm{pH}$ to activate the formation of the aryl radical. Then, $200 \mu \mathrm{L}$ of an aqueous solution of 4-aminophenyldiazonium tetrafluoroborate $(0.213 \mathrm{M})$ were added. The mixture was vigorously stirred to promote the aryl radical attachment onto the $\mathrm{SiO}_{2}$. After $1 \mathrm{~h}$ under stirring, five cycles of centrifugation/redispersion in water and ethanol were performed to remove the excess of unreacted aryldiazonium salt. After several cycles, the supernatant color changed from brown (indicating the presence of the aryldiazonium salt) to colorless. The color of $\mathrm{SiO}_{2}$ particles changed from white (pure silica) to a slight brown indicating light absorption by the covalently attached molecules.

\begin{tabular}{lc}
\hline Metal oxide particles & Mass $(\mathrm{mg}) \mathrm{X}=$ \\
\hline $\mathrm{SiO}_{2}$ & 31.4 dispersed in $500 \mu \mathrm{L}$ of water \\
$\mathrm{Gd}_{2} \mathrm{O}_{3}$ & 40 \\
$\mathrm{TiO}_{2}$ & 3 \\
$\mathrm{Al}_{2} \mathrm{O}_{3}$ & 50 \\
\hline
\end{tabular}

Dye Labeling of Metal Oxide Particles with a Two-Steps Process: $500 \mu \mathrm{L}$ of the surface-modified metal oxide particles suspension were diluted in $3 \mathrm{~mL}$ of absolute ethanol and $500 \mu \mathrm{L}$ of $2.8 \times 10^{-3} \mathrm{M}$ solution of RITC solution was added. The mixture was stirred overnight and washed by repeating centrifugation/redispersion cycles with water to remove the excess of dye. The obtained dispersion showed a slight purple coloration indicating the success of the grafting.

Dye Labeling of Metal Oxide Particles with a One-Step Process (Phenyldiazonium Salt Linked to RITC): Grafting between 4-aminophenyldiazonium tetrafluoroborate and the RITC was achieved by an addition reaction of the isothiocyanate group of the dye with the amino group of the diazonium salt. An equimolar mixture of RITC previously diluted in ethanol and an aqueous solution of the diazonium salt was stirred overnight. To dye label the metal oxide particles, $x \mathrm{mg}$ of metal oxide particles were diluted in $1 \mathrm{~mL}$ of ethanol and $1 \mathrm{~mL}$ of deionized water (mass are detailed in the previous table). $200 \mu \mathrm{L}$ of ammonia solution was then added in order to obtain a basic pH. $500 \mu \mathrm{L}$ of the RITC-phenyldiazonium solution was introduced in the mixture under stirring for $1 \mathrm{~h}$. Different cycles of centrifugation/redispersion in water were performed to remove the excess of fluorescent aryl diazonium.

Characterizations: ATR-IR spectra were measured with a BrukerVertex spectrometer over the range of $400-5000 \mathrm{~cm}^{-1}$ with a $4 \mathrm{~cm}^{-1}$ spectral resolution. Before the measurements, the samples were dried at $60{ }^{\circ} \mathrm{C}$ overnight. For easier comparison with traditional IR-spectra, the measured reflectance spectra were numerically converted to transmission spectra using the instruments software (Bruker Opus 7).

Fluorescence characterizations were performed with a Nikon AlR MP LSCM. A 100x objective (oil immersion) was used. The excitation wavelength was $561.5 \mathrm{~nm}$. $100 \mu \mathrm{L}$ of particles dispersion was deposited between two glass substrates separated by double-sided tape. The microscope was equipped with a 32-channel spectral detector allowing wavelength-resolved imaging measurements with 10-nm spectral resolution (inset Figure 3b).

\section{Supporting Information}

Supporting Information is available

\section{Acknowledgements}

This project was financially supported by the Swiss National Science Foundation, Project Nr. 132736, and the Adolphe Merkle Foundation. The authors thank Dr. Jakub Haberko for his help with the ATR-IR measurements.

[1] W. C. W. Chan, D. J. Maxwell, X. Gao, R. E. Bailey, M. Han, S. Nie, Curr. Opin. Biotechnol. 2002, 13, 40.

[2] C.-W. Lai, Y.-H. Wang, C.-H. Lai, M.-J. Yang, C.-Y. Chen, P.-T. Chou, C.-S. Chan, Y. Chi, Y.-C. Chen, J.-K. Hsiao, Small 2008, 4, 218.

[3] C. Louis, R. Bazzi, C. A. Marquette, J.-L. Bridot, S. Roux, G. Ledoux, B. Mercier, L. Blum, P. Perriat, O. Tillement, Chem. Mater. 2005, 17, 1673.

[4] M. Wu, J. W. Roberts, M. Buckley, Exp. Fluids 2005, 38, 461.

[5] B. O'Regan, M. Grätzel, Nature 1991, 353, 737.

[6] A. Van Blaaderen, A. Vrij, Langmuir 1992, 8, 2921.

[7] F. Tronc, M. Li, J. Lu, M. A. Winnik, B. L. Kaul, J.-C. Graciet, J. Polym. Sci., Part. A: Polym. Chem. 2003, 41, 766.

[8] M. Beija, M.-T. Charreyre, J. M. G. Martinho, Prog. Polym. Sci. 2011, $36,568$.

[9] G. Decher, Science 1997, 277, 1232.

[10] F. Caruso, Chem. Eur. J. 2000, 6, 413.

[11] K. T. Thurn, T. Paunesku, A. Wu, E. M. B. Brown, B. Lai, S. Vogt, J. Maser, M. Aslam, V. Dravid, R. Bergan, G. E. Woloschak, Small 2009, 5, 1318.

[12] I. Rěhoř, V. Vilímová, P. Jendelová, V. Kubícěk, D. Jirák, V. Herynek, M. Kapcalová, J. Kotek, J. Cèrný, P. Hermann, I. Lukeš, J. Med. Chem. 2011, 54, 5185.

[13] T. Paunesku, T. Rajh, G. Wiederrecht, J. Maser, S. Vogt, N. Stojičević, M. Protić, B. Lai, J. Oryhon, M. Thurnauer, G. Woloschak, Nat. Mater. 2003, 2, 343.

[14] F. M. Christensen, H. J. Johnston, V. Stone, R. J. Aitken, S. Hankin, S. Peters, K. Aschberger, Nanotoxicology 2011, 5, 110.

[15] J. L. Bahr, J. Yang, D. Kosynkin, M. J. Bronikowski, R. E. Smalley, J. M. Tour, J. Am. Chem. Soc. 2001, 123, 6536.

[16] L. Laurentius, S. R. Stoyanov, S. Gusarov, A. Kovalenko, R. Du, G. P. lopinski, M. T. McDermott, ACS Nano 2011, 5, 4219.

[17] N. Griffete, F. Herbst, J. Pinson, S. Ammar, C. Mangeney, J. Am. Chem. Soc. 2011, 133, 1646.

[18] A. Mesnage, M. A. Magied, P. Simon, N. Herlin-Boime, P. Jégou, G. Deniau, S. Palacin, J. Mater. Sci. 2011, 46, 6332. 
[19] K. Boukerma, M. M. Chehimi, J. Pinson, C. Blomfield, Langmuir 2003, 19, 6333.

[20] P. Doppelt, G. Hallais, J. Pinson, F. Podvorica, S. Verneyre, Chem. Mater. 2007, 19, 4570.

[21] D.-e. Jiang, B. G. Sumpter, S. Dai, J. Am. Chem. Soc. 2006, 128, 6030.

[22] S. Maldonado, T. J. Smith, R. D. Williams, S. Morin, E. Barton, K. J. Stevenson, Langmuir 2006, 22, 2884.

[23] A. Adenier, N. Barré, E. Cabet-Deliry, A. Chaussé, S. Griveau, F. Mercier, J. Pinson, C. Vautrin-UI, Surf. Sci. 2006, 600, 4801.

[24] A. Merson, Th. Dittrich, Y. Zidon, J. Rappich, Y. Shapira, Appl. Phys. Lett. 2004, 85, 1075.
[25] B. L. Hurley, R. L. J. McCreery, Electrochem. Soc. 2004, 151, 252-259.

[26] N. A. M. Verhaegh, A. van Blaaderen, Langmuir 1994, 10, 1427.

[27] N. C. Zhang, E. Y Ding, X. Feng, Y. Xu, H. H. Cai, Colloids Surf. B 2012, 89, 133.

[28] S. Mahouche-Chergui, S. Gam-Derouich, C. Mangeney, M. M. Chehimi, Chem. Soc. Rev. 2011, 40, 4143.

[29] B. L. Hurley, R. L. McCreery, J. Electrochem. Soc. 2004, 151, B252.

[30] M. Joselevich, F. J. Williams, Langmuir 2008, 24, 11711.

[31] W. Stöber, A. Fink, E. Bohn, J. Colloid Interface Sci. 1968, 26, 62. 